\title{
Hepatocellular Carcinoma: Updates to Screening and Diagnosis
}

\author{
Presented by Anne M. Covey, MD
}

\begin{abstract}
In what is considered to be "a global problem," liver cancer has tripled in incidence in the United States over the past 20 to 30 years, and is now present in 7 per 100,000 Americans. Thus, screening for disease should be at the forefront to effectively treat high-risk populations. At the 2018 NCCN 23rd Annual Conference, Dr. Anne M. Covey discussed the updated NCCN Guidelines for the screening and diagnosis of hepatocellular carcinoma, which place ultrasound as the most cost-effective and least toxic primary screening option, with a screening interval of approximately 6 months for individuals considered to be at high risk.

J Natl Compr Canc Netw 2018;16(5.5):663-665 doi: 10.6004/jnccn.2018.0052
\end{abstract}

\begin{abstract}
"When we perform screening, we're trying to find disease at a stage where treatment is potentially curative, and screening results in improved survival at 1,3 , and 5 years," stated Anne M. Covey, MD, Professor of Radiology, Memorial Sloan Kettering Cancer Center, and a member of the NCCN Guidelines Panel for Hepatobiliary Cancers (HCC). In her discussion at the NCCN 23rd Annual Conference, Dr. Covey not only outlined populations at high risk for HCC but also endorsed the selective use of ultrasound as a preliminary screening tool for early-stage disease.
\end{abstract}

\section{A Global Problem}

HCC is a common disease with substantial morbidity, which begs for more cost-effective and less-toxic screening options. In what is considered to be "a global problem," liver cancer is a disease that now affects 7 per 100,000 Americans and has increased in incidence 3 -fold in the United States over the past 20 to 30 years. ${ }^{2-4}$ This rise in incidence can be accounted for by an increase in immigration from epidemic areas, development of cirrhosis in patients who were infected with

\footnotetext{
Presented by Anne M. Covey, MD, Memorial Sloan Kettering Cancer Center, New York, New York.

Dr. Covey has disclosed that she has served as a scientific advisor for Accurate Medical, and that she has equity interest/stock options in Amgen Inc.

Correspondence: Anne M. Covey, MD, Memorial Sloan Kettering Cancer Center, 1275 York Avenue, New York, NY 10065.

Email: coveya@mskcc.org
}

hepatitis C virus (HCV) in the 1970s and 1980s, as well as the increase in nonalcoholic fatty liver disease. ${ }^{2}$ As Dr. Covey pointed out, the incidence of disease parallels that of survival, which is $<20 \%$ is 5 years.

In countries that heavily screen high-risk populations for liver cancer, overall survival (OS) has improved. ${ }^{5}$ According to Dr. Covey, this may be "coincident with implementation of national screening programs, which have been in place since the late 1990s."

A similar study conducted in the Netherlands that examined all patients diagnosed with HCC between 2005 and 2012, "found that just over a quarter of patients had been screened, and those who were screened had smaller disease at the time of detection: $2.6 \mathrm{~cm}$ versus $6 \mathrm{~cm}$." Screening has come to be the most effective way to detect early disease and increase survival. In reference to the Netherlands study, Dr. Covey stated, "The results showed significantly prolonged survival in patients who were screened compared with those whose disease was detected when it was evident based on signs or symptoms."

\section{High-Risk Populations}

As Dr. Covey made clear, most patients who develop HCC do so in the setting of either chronic hepatitis B (HBV) or cirrhosis of any cause including but not limited to $\mathrm{HBV}, \mathrm{HCV}$, or alcoholic or nonalcoholic fatty liver disease. ${ }^{8-10}$ It is important to note that in the setting of chronic $\mathrm{HBV}, 30 \%$ to $50 \%$ of patients develop $\mathrm{HCC}$ without underlying cirrhosis. 
Selective screening for HCC is most applicable, because patients at high risk can be identified to detect disease in its early stages, when more curative options are available. Roughly $80 \%$ of patients with HCC have a known risk factor, ${ }^{11}$ and we believe that implementing screening programs for those known populations improves OS. Other risk factors in the setting of HCV cirrhosis include male sex, coinfection with HIV or HBV, older age, diabetes, and obesity. ${ }^{10}$

\section{Emphasis on Screening: Focus on Ultrasound}

Dr. Covey emphasized the importance of screening patients at risk to identify disease at its preclinical stages. In the 2018 updated NCCN Guidelines for HCC, patients who are at risk should undergo ultrasound with or without assessment of alpha-fetoprotein (AFP) as a primary screening tool. ${ }^{12}$ Dr. Covey noted that "ultrasound is probably not the most sensitive test, but data support its use" in screening those at risk for HCC. "The benefits of ultrasound are that it is inexpensive, widely available, and there is no radiation. When we apply this tool in a center that does a lot of screening, sensitivity is around $85 \%$, which is pretty high, and the specificity is as high or higher." Exceptions to screening, according to Dr. Covey, would include patients not eligible for treatment, such as those with Child-Pugh C disease not on a transplant waiting list and those with comorbidities that preclude treatment.

According to the 2018 NCCN Guidelines, which are in line with those from LI-RADS (Liver
Imaging Reporting and Data System), for patients with a negative ultrasound or a nodule $<10 \mathrm{~mm}$, repeat ultrasound and AFP evaluation in 6 months is indicated. In those with a positive AFP test or a nodule $\geq 10 \mathrm{~mm}$ on ultrasound and capsular retraction or vascular invasion, further workup with CT or MRI would be required (Figure 1).

In the United States, Dr. Covey noted that CT and MRI seem to be used more often than ultrasound for detecting HCC. In addition to the higher cost associated with CT and MRI, she mentioned that these modalities tend to detect lesions that are not HCC or are not clinically significant.

"So although we recognize that people are using CT and MRI as a screening tool, we don't recommend it as the first test." Potential limitations of ultrasound screening explored by Dr. Covey include that it is dependent on both operator ability and patient body habitus, and that some cirrhotic livers are "ultrasound unfriendly."

Dr. Covey briefly mentioned serum biomarkers, such as AFP and others. Although the use of ultrasound and AFP together may increase sensitivity by approximately $4 \%$ to $6 \%$, the combination nearly doubles the cost for each small HCC detected. ${ }^{12,13}$ "Early tumors in the United States tend not to be associated with elevated serum AFP," revealed Dr. Covey, "so in the 2018 Guidelines, this is considered optional and should only be used in conjunction with ultrasound."

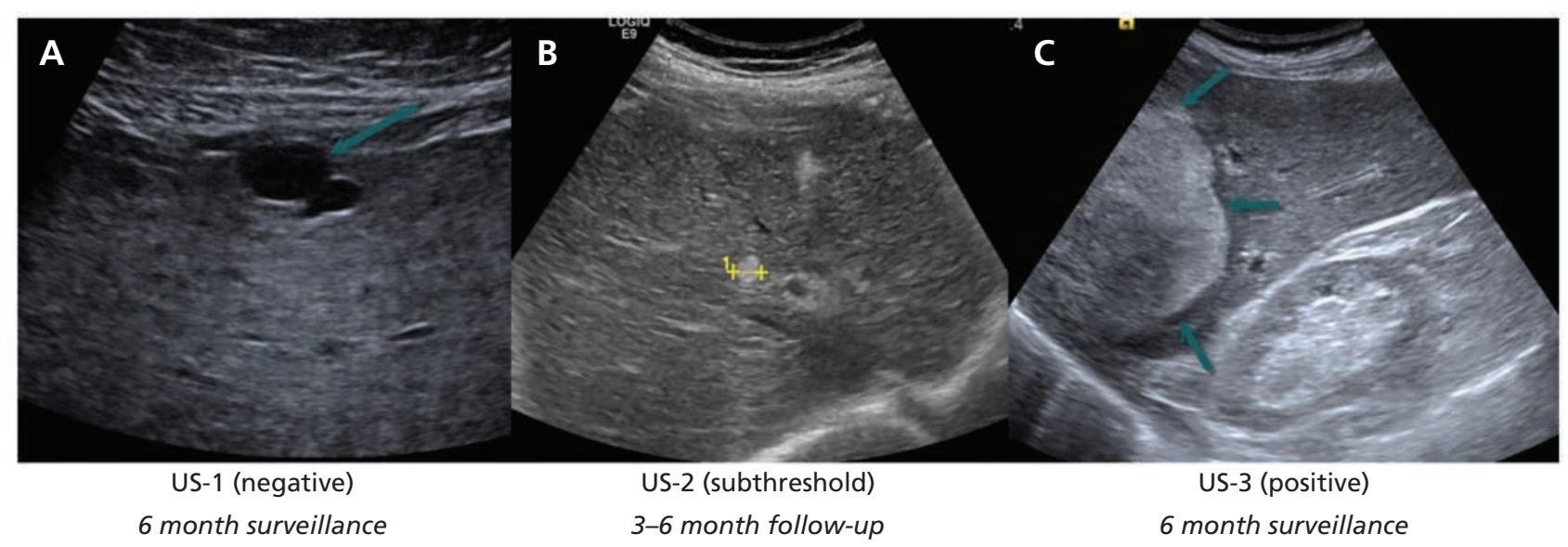

Figure 1. Ultrasound (US) LI-RADS decision tree. (A) A hypoechoic lesion with a thin wall and enhanced through transmission is diagnostic of a cyst; this is an example of an US-1 or a negative screening study. (B) In another patient, a hyperechoic lesion measuring $7 \mathrm{~mm}$ is identified. Because the lesion is $<10 \mathrm{~mm}$, it is below the threshold for CT or MR. Thus, follow-up US in 3 to 6 months is recommended. (C) A hyperechoic liver mass $>10 \mathrm{~mm}$ is suspicious for hepatocellular carcinoma and warrants further evaluation with multiphase CT or MR. 


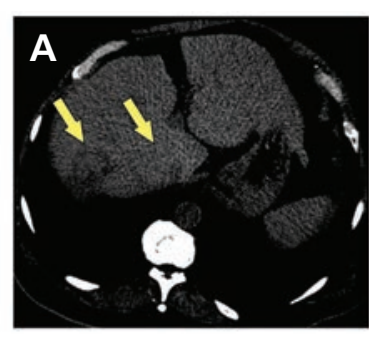

Non-contrast

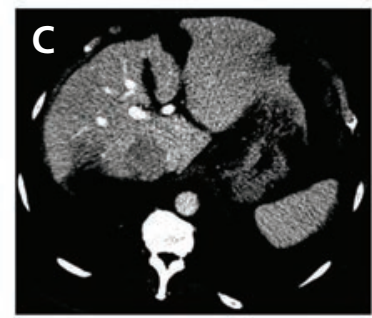

Portal venous phase

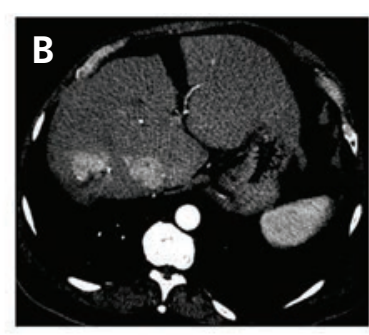

Arterial phase

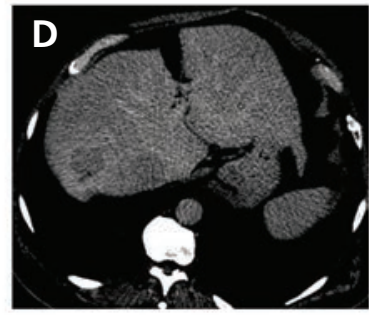

Delayed phase
Figure 2. Classic appearance of hepatocellular carcinoma (HCC) on multiphase CT. (A) On non-contrast images, 2 hypoechoic lesions (arrows) are seen in the right liver of a patient with hepatitis $C$ cirrhosis. (B) On arterial phase, there is brisk enhancement of both lesions, which demonstrate wash-out on (C) portal venous and (D) delayed images. This constellation of findings in a patient with a known risk factor is diagnostic of HCC.

\section{Screening Intervals}

"Six months seems to be the appropriate screening interval," Dr. Covey stated, because the "doubling time for most HCCs is in the 150- to 200-day range." Several studies have examined and compared less frequent ${ }^{14}$ and more frequent ${ }^{15}$ screening intervals. Although a randomized controlled trial of patients with alcoholic cirrhosis screened at 3 versus 6 months did not increase the detection of small lesions, it did lead to detection of more focal lesions, which proved to be nonmalignant on further workup. ${ }^{14}$ In an Italian study that compared 6 - and 12 -month surveillance, ${ }^{15}$ the researchers found that 6 -month surveillance increased detection of HCC amenable to curative treatment and improved OS (45 vs 30 months, respectively).

\section{Ability to Treat Once Detected}

Dr. Covey emphasized that HCC is one of very few tumors that can be diagnosed by imaging alone.

The Barcelona Clinic Liver Cancer (BCLC) staging system is a useful tool that uses tumor characteristics and liver dysfunction to guide treatment decisions, Dr. Covey commented. When screening detects an early stage of disease in a patient with a good performance status, curative treatments yield a 5-year survival rate between $50 \%$ and $70 \%$. "That is quite excellent for HCC," she admitted. If the disease is detected at a later stage in patients who have a compromised performance status, metastatic disease, or vascular involvement, "their outcomes are significantly worse," stated Dr. Covey, with 3-year survival rates between $10 \%$ and $40 \%$.

Once a lesion is detected, the NCCN Guidelines indicate that abdominal multiphasic CT or MRI is the next step to confirm the diagnosis (Figure 2). The "Principles of Imaging" criteria (available at NCCN. org) include that a multidetector scanner should be used for CT and MRI imaging and there should be multiple phases of imaging, including arterial, portal, and delayed. "Without arterial phase enhancement and a delayed washout, you cannot make a diagnosis of HCC based on imaging alone," added Dr. Covey.

\section{References}

1. Zhang B, Yang B, Tang Z. Randomized controlled trial of screening for hepatocellular carcinoma. J Cancer Res Clin Oncol 2004;130:417-422.

2. Petrick JL, Braunlin M, Laversanne $M$, et al. International trends in liver cancer incidence, overall and by histologic subtype. Int J Cancer 2016;139:1534-1545.

3. Yang JD, Harmsen WS, Slettedahl SW, et al. Factors that affect risk for hepatocellular carcinoma and effects of surveillance. Clin Gastroenterol Hepatol 2011;9:617-623.e1.

4. Heimbach JK, Kulik LM, Finn RS, et al. AASLD guidelines for the treatment of hepatocellular carcinoma. Hepatology 2018;67:358-380.

5. Park JW, Chen M, Colombo M, et al. Global patterns of hepatocellular carcinoma management from diagnosis to death: the BRIDGE Study. Liver Int 2015;35:2155-2166.

6. Bertuccio P, Turati F, Carioli G, et al. Global trends and predictions in hepatocellular carcinoma mortality. J Hepatol 2017;67:302-309.

7. van Meer S, de Man RA, Coenraad MJ, et al. Surveillance for hepatocellular carcinoma is associated with increased survival: results from a large cohort in the Netherlands. J Hepatol 2015;63:1156-1163.

8. Parkin DM, Bray F, Ferlay J, et al. Global cancer statistics, 2002. CA Cancer J Clin 2005;55:74-108.
9. Yang HI, Yuen MF, Chan HL, et al. Risk estimation for hepatocellular carcinoma in chronic hepatitis B (REACH-B): development and validation of a predictive score. Lancet Oncol 2011;12:568-574.

10. Lee MH, Lu SN, Yuan Y, et al. Development and validation of a clinical scoring system for predicting risk of HCC in asymptomatic individuals seropositive for anti-HCV antibodies. PLoS One 2014;9:e94760.

11. Fattovich G, Stroffolini T, Zagni I, Donato F. Hepatocellular carcinoma in cirrhosis: incidence and risk factors. Gastroenterology 2004;127(Suppl 1):S35-50.

12. Fetzer DT, Rodgers SK, Harris AC, et al. Screening and surveillance of hepatocellular carcinoma: an introduction to ultrasound liver imaging reporting and data system. Radiol Clin North Am 2017;55:1197-1209.

13. Sherman M, Colombo M. Hepatocellular carcinoma screening and diagnosis. Semin Liver Dis 2014;34:389-397.

14. Trinchet JC, Chaffaut $C$, Bourcier V, et al. Ultrasonographic surveillance of hepatocellular carcinoma in cirrhosis: a randomized trial comparing 3and 6-month periodicities. Hepatology 2011;54:1987-1997.

15. Santi V, Trevisani F, Gramenzi A, et al. Semiannual surveillance is superior to annual surveillance for the detection of early hepatocellular carcinoma and patient survival. J Hepatol 2010;53:291-297. 